CASO CLÍNICO

\title{
Adenoma del pezón: reporte de un caso
}

\section{Nipple adenoma: Case report}

Yesika J. Dávila-Zablah*, Adrián Barrios-Ruiz, Gabriela S. Gómez-Macías y Margarita L. Garza-Montemayor

Centro de Imagen Diagnóstica, Hospital San José, Tecnológico de Monterrey, Monterrey, Nuevo León, México

\section{RESUMEN}

El adenoma de pezón representa una proliferación benigna poco común de los conductos mamarios. Clínicamente puede confundirse con la enfermedad de Paget e histopatológicamente con carcinoma o lesiones precursoras. Por ello, es esencial el estudio histológico y el análisis de inmunofenotipo para su diagnóstico diferencial. El presente estudio describe las características de un caso clínico de adenoma del pezón, así como la revisión de la literatura.

Palabras clave: Adenoma del pezón. Tumor benigno de mama. Papilomatosis florida del pezón. Células mioepiteliales. Inmunofenotipo. Adenomatosis. Adenoma papilar.

\section{ABSTRACT}

Nipple adenoma is a very uncommon, benign proliferative process of lactiferous ducts of the nipple. Clinically mimic the presentation of mammary Paget's disease and histopathologically with carcinoma or precursor lesions. Therefore, the histological and immunophenotypic analysis is essential for the differential diagnosis. The present study describes the characteristics of a clinical case of Nipple adenoma, as well as review of the literature.

Key words: Nipple adenoma. Benign breast tumor. Florid nipple papillomatosis. Myoepithelial cells. Immunophenotype. Adenomatosis. Papillary adenoma. 


\section{INTRODUCCIÓN}

El adenoma del pezón es un tumor epitelial benigno poco común que se origina de los conductos lactóforos del pezón. Ocurre generalmente de forma unilateral y en la cuarta década de la vida. Esta entidad se puede confundir clínicamente con la enfermedad de Paget e histológicamente con el carcinoma de mama. Es importante su análisis morfológico asociado a un panel de inmunofenotipo para reconocer esta lesión y diferenciarla de una entidad maligna. En el presente estudio se describe un caso de un adenoma del pezón, sus hallazgos radiológicos y patológicos, así como revisión de la literatura.

\section{CASO CLÍNICO}

Paciente de sexo femenino de 28 años de edad, que inicia su padecimiento 3 meses antes a su estudio, con telorrea sanguinolenta, además de hipersensibilidad en el pezón derecho y una tumoración palpable de $1 \mathrm{~cm}$ (Fig. 1).

Se realiza citología, y se reporta papiloma con atipia. Posteriormente se realiza un ultrasonido mamario bilateral, en el que se observa el tejido mamario de eco textura homogénea de predominio fibroglandular. En la mama derecha, en la región retroareolar, se observan 2 nódulos sólidos intraductales de 2 y $6 \mathrm{~mm}$, uno de ellos con vascularidad a la aplicación Doppler color. Adicionalmente se toma una proyección mediolateral oblicua (MLO), sin encontrar hallazgos de interés. Se clasifica como BI-RADS 4A y se sugiere realizar biopsia (Figs. 2 y 3).

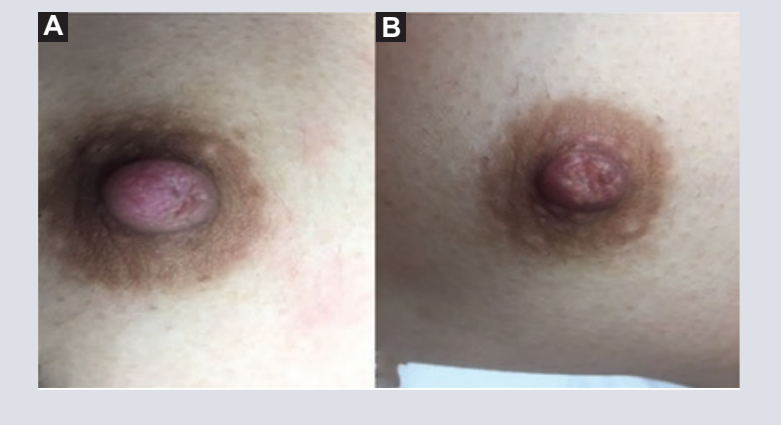

Figura 1. A y B: Fotografía de la paciente en donde se observan cambios en el pezón derecho, con presencia de una pequeña tumoración palpable.

Posteriormente se realiza una resonancia magnética simple con contraste, en la que se observa el tejido mamario de predominio fibroglandular, con realce de fondo leve posterior a la administración de contraste y un nódulo intrapezón hiperintenso en T2 e hipercaptante, mide $1 \mathrm{~cm}$, muestra realce en anillo con curva dinámica en meseta (tipo 2) con restricción a la difusión, con un valor de ADC bajo, $0.6 \times 10^{-3} \mathrm{~mm}^{2} / \mathrm{s}$. Sugiere corresponder con papiloma. Se caracteriza BIRADS 4 y se recomienda biopsia (Fig. 4).

Se realiza biopsia escisional, y se observa una lesión central al pezón con erosión de la epidermis, proliferada, de apariencia compleja, datos en relación con un adenoma del pezón. Presenta ductos distendidos con proliferación de epitelio e hiperplasia atípica, con luces rígidas y en hendidura, núcleos con atipia, así como hiperplasia de células columnares (Fig. 5).

\section{DISCUSIÓN}

El adenoma de pezón es un tumor epitelial benigno poco frecuente de los conductos, que se origina del complejo areola-pezón ${ }^{1-20}$. 


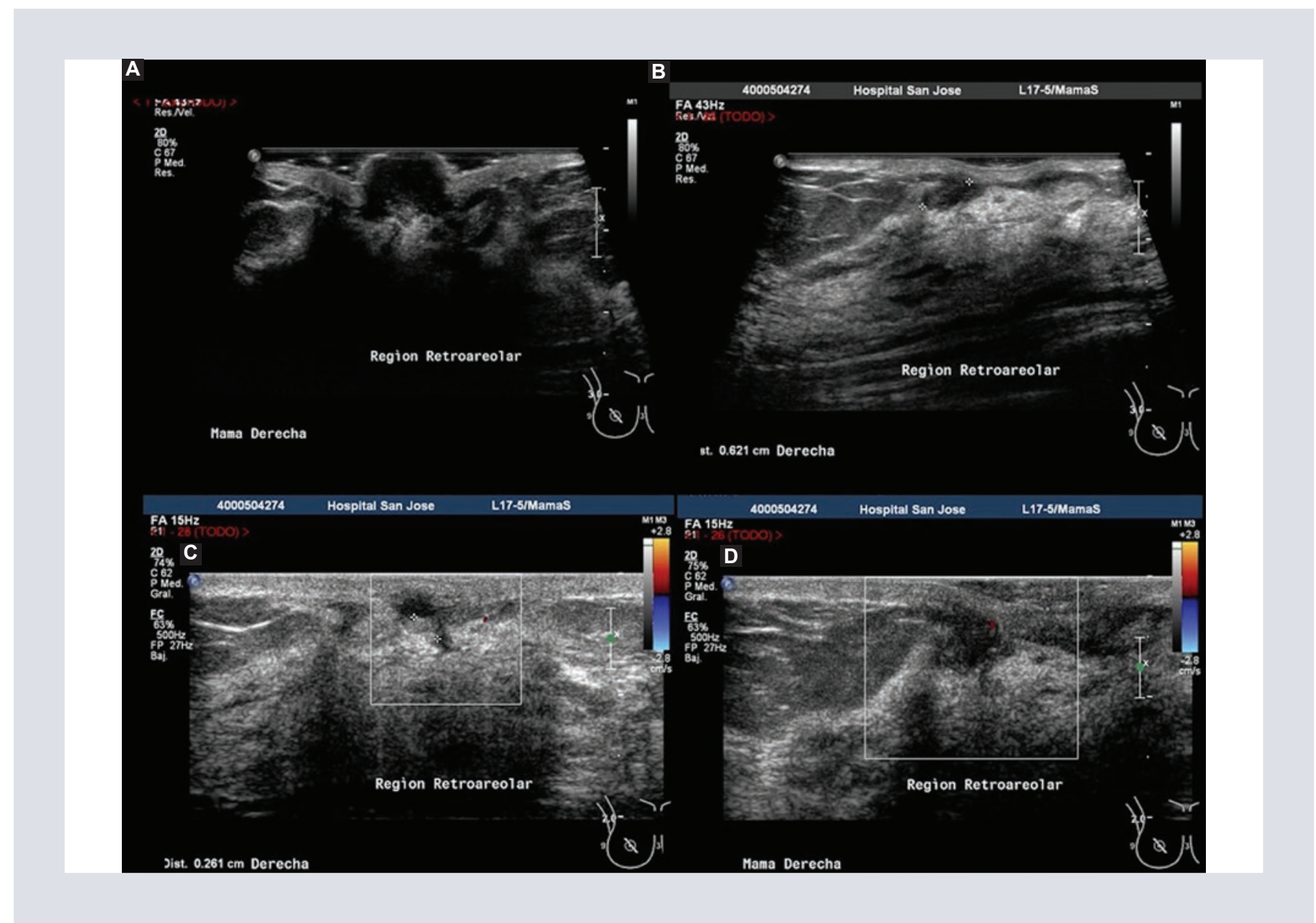

Figura 2. Ultrasonido mamario. A y B: Se observan en la región retroareolar derecha 2 nódulos sólidos intraductales de 2 y 6 mm; C y D: Muestran vascularidad periférica a la aplicación Doppler color.

Ocurre generalmente de forma unilateral en pacientes en la cuarta década de la vida $^{1,3,5,7,12,13,16-20}$, es raro en hombres y adolescentes ${ }^{1,3,7,13,17,20}$. La paciente del presente estudio tiene 28 años, edad inferior a lo reportado en la literatura, y tal como se describe en diferentes estudios se presenta clínicamente de forma unilateral.

La lesión es conocida también como adenoma erosivo, adenomatosis papilar superficial, papilomatosis ductal subareolar o papilomatosis florida ${ }^{1,2,5,7-9,13,17,18}$.

Descrito por primera vez por Haagensen, et al., en 1951, con el nombre de papiloma intraductal benigno ${ }^{19,20}$. Jones, en 1955, lo describe como una papilomatosis florida $\mathrm{y}$, posteriormente, en 1989, como adenoma $3,5,11,13,16-18$. También reportado por Rosen en 198311,12.

Representa el 15\% de los tumores en el pezón 7 .

Es comúnmente confundido con la enfermedad de Paget y, en ocasiones, es difícil diferenciarlo de un carcinoma de mama ${ }^{1-7,9,10,13,16,19}$.

Los síntomas iniciales son erosión del pezón, inflamación, eritema y descarga serosa o serosanguinolenta en un 60 a $70 \%$ de los casos. En etapas avanzadas, el pezón aparece aumentado de tamaño, engrosado e indurado 


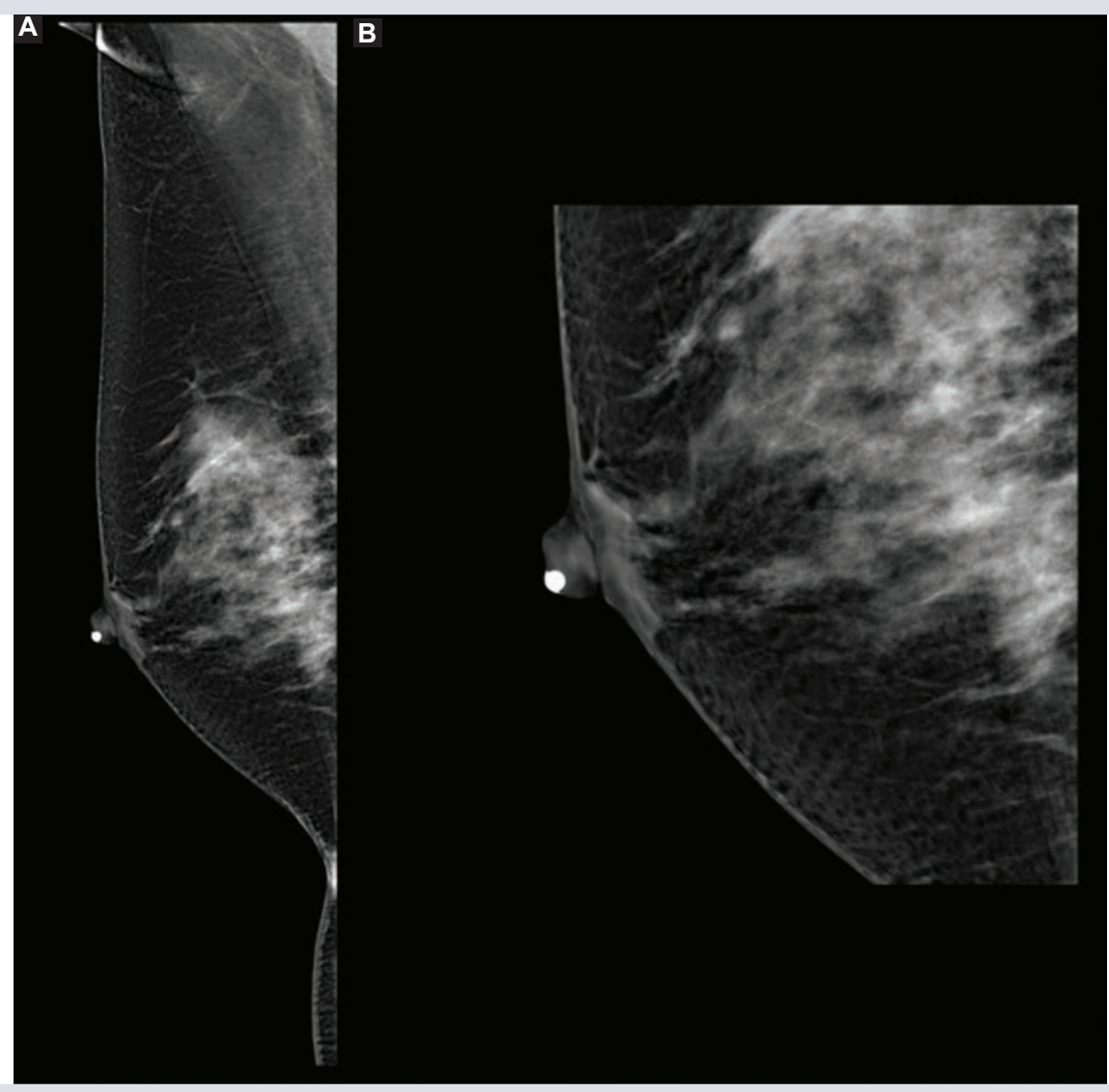

Figura 3. Mamografía. A: Proyección mediolateral oblicua (MLO); B: Magnificación digital de la mama derecha, no se observan distorsiones ni calcificaciones sospechosas de malignidad.

o, en ocasiones, como una masa subareolar palpable $1-3,5-12,13,15-20$. Si esta condición progresa, puede complicarse y formar abscesos o adenopatías $^{7,18}$.

Nuestra paciente presenta telorrea sanguinolenta, hipersensibilidad en el pezón y tumoración palpable, lo cual es concordante con las publicaciones existentes.

Debido al tamaño, generalmente no se detecta en una mamografía ni en un ultrasonido. Algunos de los hallazgos mamográficos encontrados son una masa pequeña oval en el pezón o como asimetría en el tamaño del pezón $^{7,8,10}$. Encontrar calcificaciones es poco común ${ }^{8,11}$. Nuestra paciente no presenta ningún hallazgo mamográfico positivo.

En el ultrasonido se detecta una masa lobulada u oval, homogénea, iso o hipoecoica en el pezón, con incremento en la vascularidad a la aplicación Doppler color ${ }^{8,11}$, tal como se presenta en nuestra paciente.

La resonancia magnética reporta reforzamiento anormal del pezón con curva persistente o de lavado rápido, no se observan zonas hipertensas en $\mathrm{T} 2^{7,8,10,14}$. La resonancia de nuestra paciente presenta un nódulo intrapezón hipercaptante, con curva dinámica en meseta. 

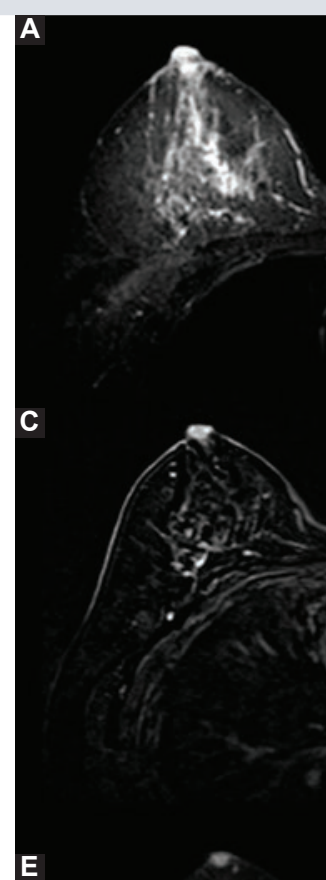

E

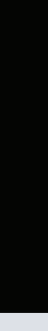

B
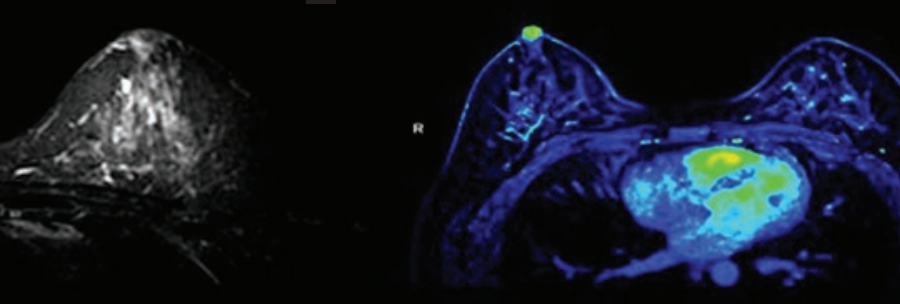

D

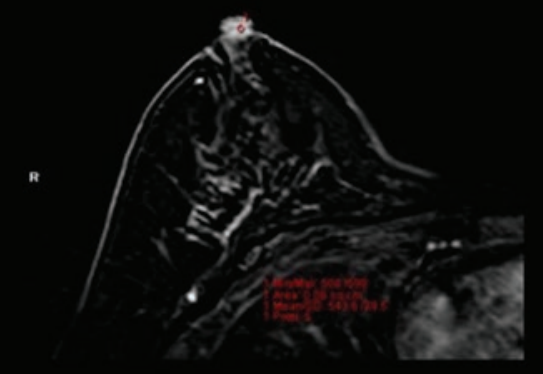

$\mathbf{F}$

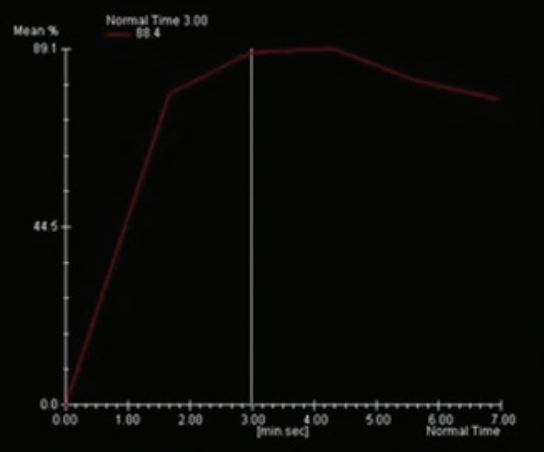

Figura 4. Resonancia magnética con contraste. A: Se observa un nódulo intrapezón hiperintenso en T2; B y C: Después de la administración de contraste muestra realce en anillo; D, E y F: Con curva dinámica en meseta (tipo 2).

Histopatológicamente es un tumor heterogéneo, debido a sus formas variadas de crecimiento y asociación, se han descrito 4 patrones: a) patrón papilomatoso esclerosante, frecuentemente indistinguible del papiloma esclerosante; b) patrón papilomatoso, hiperplasia papilar florida del epitelio ductal; c) patrón de adenosis que evidencia hiperplasia mioepitelial, y d) patrón mixto proliferativo, que es la combinación de los 3 patrones (metaplasia de los conductos con quistes, metaplasia apocrina y acantosis del epitelio) $)^{1,13}$.

Se requiere de un análisis morfológico delicado y panel de inmunofenotipo para su diagnóstico diferencial con otras neoplasias malignas de la mama, para evitar una mastectomía no indicada, por lo que se recomienda la escisión ${ }^{1-3}$.

El estudio anatomopatológico es fundamental para el diagnóstico, muestra una lesión circunscrita, no encapsulada a nivel dérmico de estructuras tubulares circulares, ovales o irregulares, que se extienden hacia abajo desde la epidermis. Estas estructuras tubulares presentan una doble capa de células epiteliales característica: una capa luminal de células mioepiteliales cuboidales 

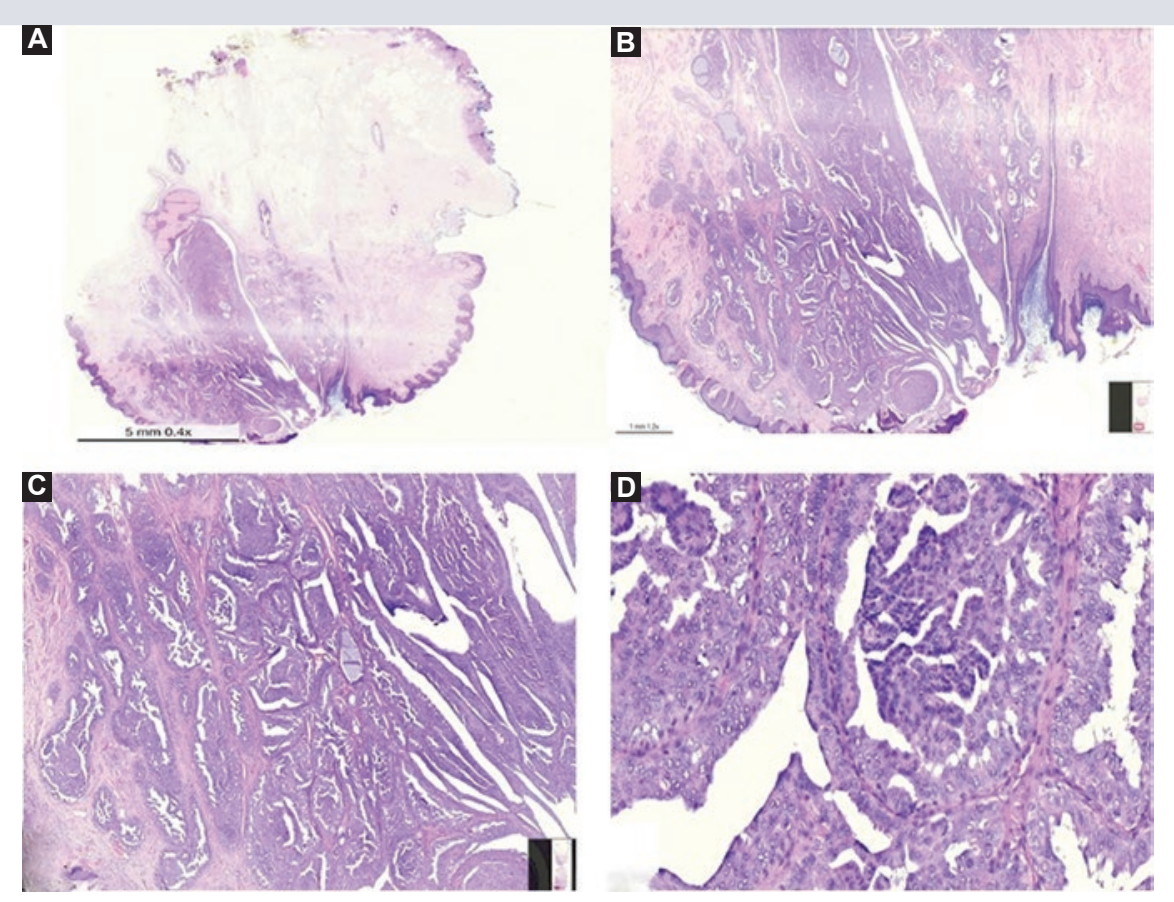

Figura 5. Imágenes de anatomía patológica. A: Panorámica de la lesión, que se encuentra central al pezón, con erosión de la epidermis; B: Vista a $5 X$, donde se aprecia una lesión proliferada, de apariencia compleja, con áreas de hiperplasia; C: Vista 10X los ductos se encuentran distendidos, con proliferación del epitelio; D: Vista 40X se aprecia hiperplasia atípica, con presencia de luces rígidas y en hendidura, núcleos con atipia, así como hiperplasia de células columnares.

y una capa luminal de células cilíndricas que pueden mostrar proyecciones secretoras en su borde luminal, que a veces pueden ser muy pronunciadas y ocupar casi toda la $\operatorname{luz}^{1,3,5,6,11,13,17,16,19}$.

Los túbulos pueden estar ocupados por un material eosinofílico, aparentemente secretado por las células luminales. Puede encontrarse erosión y ulceración y quistes de queratina, la epidermis puede mostrar hiperqueratosis y acantosis ${ }^{19}$.

Además de la enfermedad de Paget, dentro de sus diagnósticos diferenciales por patología, se deben incluir el carcinoma ductal in situ de bajo grado, carcinoma tubular, adenoma siringomatoso infiltrativo y papiloma central subareolar solitario'.
Distinguir un adenoma de un papiloma intraductal puede ser difícil en el frotis, la secreción del pezón en un papiloma intraductal muestra principalmente grupos papilares de células ductales que muestran conversión de células focales de espuma y conversión de células fusiformes, estas características no se ven en el adenoma del pezón ${ }^{2}$.

Los hallazgos histopatológicos que lo diferencian de un adenocarcinoma de mama de bajo grado de malignidad son ausencia de atipia citológica, los dos tipos de células del epitelio tubular, la papilomatosis intraductal, la simetría, la buena delimitación y la localización superficial en el tejido subareolar ${ }^{16,19}$.

El uso de p63 es para diferenciarlo de un cáncer in situ, en el adenoma no se expresa 
el p63 o aparece de forma discontinua. El CK 5/6 junto con las células mioepiteliales se presenta en una lesión de proliferación epitelial $^{1,3}$.

Se han reportado algunos casos de presentación bilateral y también asociado con carcinoma maligno, por lo que existe la probabilidad de que el adenoma desarrolle un carcinoma hasta en un $1.2 \%$, sin embargo, no existen datos suficientes en la literatura ${ }^{1,2,5,7,13}$.

El tratamiento de elección es la escisión, sin embargo, si no es completa, puede ocurrir recurrencia hasta en un $30 \%{ }^{2-5,7-9,11-13,16-20}$ de los casos. El pronóstico es excelente ${ }^{1}$.

\section{CONCLUSIÓN}

Es importante conocer la existencia del adenoma, ya que clínicamente puede confundirse con la enfermedad de Paget. El radiólogo debe considerar esta entidad dentro de los diagnósticos diferenciales en la patología del pezón. Aunque el adenoma de pezón puede diagnosticarse con un examen citológico y biopsia de corte, se recomienza la escisión de la lesión para su adecuada caracterización histológica y de inmunofenotipo, y descartar lesiones precursoras o carcinoma invasor, así como para evitar recurrencia.

\section{CONFLICTO DE INTERESES}

Los autores declaran que no existe conflicto de intereses.

\section{RESPONSABILIDADES ÉTICAS}

Protección de personas y animales. Los autores declaran que para esta investigación no se han realizado experimentos en seres humanos ni en animales.

Confidencialidad de los datos. Los autores declaran que han seguido los protocolos de su centro de trabajo sobre la publicación de datos de pacientes.

\section{Derecho a la privacidad y consentimiento} informado. Los autores han obtenido el consentimiento informado de los pacientes y/o sujetos referidos en el artículo. Este documento obra en poder del autor de correspondencia.

\section{BIBLIOGRAFÍA}

1. Di Bonito M, Canile M, Collina F. Adenoma of the nipple: A clinicopathological report of 13 cases. Oncology Letters. 2014;7:1839-42.

2. Kijama Y, Matsukita S, Yoshinaka H. Adenoma of the Nipple: Report of a Case. Breast Cancer. 2006;13:95-99.

3. Fuji T, Yajima R, Morita H, Yamaguchi S, Tsutsumi S, Asao T, et al. Adenoma of the nipple projecting out of the nipple: curative resection without excision of the nipple. World J Surg Oncol. 2014;12:1-4.

4. Canbay E, Aydogan F, Baghaki S, lvan I, Gazioglu E. Nipple Adenoma of the Breast: Reconstruction of Nipple from the areola. Nobel Medicus. 2010, $8: 3: 127-29$.

5. Abbas A, Zaher A, Arini A, Chaudhry I. Adenoma of the Nipple, Mimicking Paget's Disease of the Breast: Report of a Case. Adv Breast Cancer Res. 2014,3:96-9.

6. Healy CE, Dijkstra B, Walsh M, Hill AD, Murphy J. Nipple Adenoma: A differential Diagnosis for Paget's Disease. Breast J. 2003,4:325-6.

7. Geffroy D, Dumoulins D. Clinical abnormalities of the nipple-areola complex: The role of imaging. Diagn Interv Imaging. 2015; 07:1-12.

8. Onifite T, Scoggins M, Dogan B. Imaging Approach of Nipple Masses: What a Radiologist Should Know. Contemporary Diagnostic Radiology. 2015; 38:25,1-8

9. Costa D, Taddese A, Cure M. Common and Unusual Diseases of the Nippleareolar complex. Radiographics. 2007;27:65-77.

10. Sarica O, Zeybek E, Ozturk E. Evaluation of Nipple-Areola Complex With Ultrasonography and Magnetic Resonance Imaging. J Comput Assit Tomogr. 2010;34(4):575-86

11. AlSharif S, Tremblay F, Omeroglu A, Altinel G, Sun S, Mesurolle B. Infiltrating Syringomatous Adenoma of the Nipple: Sonographic and Mammographic Features with Pathologic Correlation. J Clin Ultrasound. 2014;42(7):427-9. 
12. Riaz N, Massoid S, Idress R, Kayani N. Infiltrating Syringomatous Adenoma of Nipple. J Coll Physicians Surg Pak. 2008;18:7:438-9.

13. Aftab K, Idrees R. Nipple Adenoma of Breast: A Masquerader of Malignancy. J Coll Physicians Surg Pak. 2010;20(7):472-4.

14. S. Manso Garcia S. Plaza Loma S, Rodríguez de Diego Y, Zurdo de Pedro V, Pintado Garrido R Villacastin Ruiz E. Nipple-areolar complex: diagnostic challenges. European Society of Radiology, 2014; p. 1-12

15. Nicholson BT, Harvey JA, Cohen MA. Nipple-areolar complex: Normal Anatomy and benign and malignant Processes. Radiographics. 2009;29:509-23.

16. Mattar D, Romero G, Betancourt L. Adenomatosis erosiva del pezón. Rev Venz Oncol. 2006;18(2):115-9.
17. Monteagudo B, De las Heras C, Aguilera Navarro C, Used Aznar MM, García Rego JA, Álvarez JC, et al. Adenomatosis erosiva del pezón. Med Cutan Iber Lat Am. 2008;36(5):244-47.

18. Achenbach R, Pittarro E, Sanchez G. Adenomatosis Erosiva del pezón. Rev Argent Dermatol. 2008;89:208-11.

19. Vicuña C, Escalaya G, Junco ML, Chian C. Adenomatosis erosiva del pezón: reporte de un caso. Folia Dermatol Peru. 2012;23(1):21-4.

20. Lobato Miguélez J, Moreno Domingo J, Martínez Urruzola J, Arriba Olivenza T, Andía Ortiz D, López Valverde M. Adenoma de pezón: a propòsito de un caso. Clin Invest Gin Obst. 2014;41(2):89-91. 